\title{
IL-27 suppresses type 2 immune responses in vivo via direct effects on group 2 innate lymphoid cells
}

\author{
T Mchedlidze ${ }^{1}$, M Kindermann ${ }^{1}$, AT Neves ${ }^{2}$, D Voehringer ${ }^{2}$, MF Neurath ${ }^{1}$ and S Wirtz ${ }^{1}$
}

Group 2 innate lymphoid cells (ILC2) were recently characterized by their ability to produce significant amounts of type-2 signature cytokines and drive central beneficial and pathological features of type- 2 immune responses. Although factors such as IL-33 and IL-25 were shown to have ILC2 activating capacity, it is not well understood, how ILC2 responses are regulated in vivo. Here we provide compelling evidence that IL-27-signalling directly inhibits ILC2 responses and reveal a novel mechanism for negative regulation of the innate arm of type-2 immunity. We demonstrate that IL-27-deficiency is linked to increased mucosal presence of ILC2 in a model of inflammatory lung disease. Moreover, IL-27-treatment inhibited ILC2 proliferation and cytokine production and significantly reduced their accumulation in vivo. During helminth infection, regulation of ILC2 by IL-27 directly impacted anti-parasitic immunity. Thus, therapeutic modulation of the IL-27/IL-27R axis may be relevant in a number of inflammatory conditions associated with dysregulated type-2 responses.

\section{INTRODUCTION}

Innate lymphoid cells (ILCs) are a recently characterized heterogeneous but developmentally related group of lymphoidlike innate immune cells that lack specific antigen receptors. Several studies in the last years have discovered that ILCs contribute in multiple ways to protection from helminth and bacterial infections and mediate tissue repair and wound healing processes in different anatomical compartments. ${ }^{1,2}$ Among ILC group 2 innate lymphoid cells (ILC2) are considered to be a particular ILC subset that drives central features of type 2 responses in vivo. ILC2 were characterized by their ability to produce significant amounts of the type 2 signature cytokines IL-5, IL-9 and IL-13 and intrinsic expression of several transcription factors such as Ror $\alpha^{3,4}$ Gata3, ${ }^{5,6} \mathrm{Gfi1}^{7}$ and Bcl11 $\mathrm{b}^{8-10}$ has been shown to be important for their developmental program. Moreover, ILC2 are relatively abundant in tissues harboring barrier functions such as gut, lung and skin and a critical role of ILC2 and ILC2-derived cytokines in the context of host protection and tissue repair during helminthic and viral infections and for metabolic body homeostasis has been demonstrated. ${ }^{11-13}$ By contrast, dysregulated ILC2 activation was shown to promote chronic type 2 immune responses in mouse models of allergic airway hyperactivity and tissue fibrosis. ${ }^{14-16}$ Several epithelial-derived or damage-associated cytokines such as IL-33, IL-25, TSLP and TL1a are well-known for their ILC2 activating capacity. Besides, autoregulatory IL-9 production and T cell-derived IL-2 stimulation support ILC2 maintenance during immune responses. ${ }^{17,18}$ However, not much is known about immunological factors that negatively modulate ILC2 proliferation and activation during prototypic type 2 immune responses.

The heterodimeric IL-12-related cytokine IL-27 was initially described as important trigger of proinflammatory Th1 responses. More recent studies implicated this factor in the negative regulation of Th17 cells, regulatory $\mathrm{T}$ cells and myeloid cells. ${ }^{19-21}$ Moreover, in rather Th2-associated murine models of asthma or parasitic infections, IL-27R $\alpha$ deficiency resulted in exacerbated lung pathology and airway hyperresponsiveness or increased anti-parasitic immunity. ${ }^{22-24}$ While in these studies IL-27 regulation of Th2 cells was suggested to be primarily responsible for the altered disease phenotype of IL-27 R $\alpha^{-1-}$ mice, recent studies provided evidence that IL-27 as well as type I and type II interferons are able to suppress ILC2 responses in vitro and in vivo. ${ }^{25,26}$ To address the role of IL-27 signalling in

${ }^{1}$ Department of Medicine 1, University Medical Center, Friedrich-Alexander-University, Erlangen-Nuremberg, Germany and ${ }^{2}$ Department of Infection Biology, University Medical Center, Friedrich-Alexander-University, Erlangen-Nuremberg, Germany. Correspondence: S Wirtz (stefan.wirtz@uk-erlangen.de) 
the initiation phase of type 2 responses, we examined here the potential of IL-27 as regulator of innate type 2 responses in mouse models of lung inflammation and primary helminth infections. We show that IL-27 receptor signalling directly regulates ILC2 responses and that this interaction profoundly affected the extent and quality of the type 2 response in vivo. Thus, suppression of ILC2 functions by IL-27 represents a novel mechanism for the negative regulation of the innate arm of type 2 immunity.

\section{RESULTS}

\section{Lung accumulation of ILC2 cells in type 2 immune responses is increased in the absence of IL-27}

Although IL-27 has been recognized as a regulator of adaptive immune responses for several years now, its function in controlling innate immunity remains to be fully characterized. Interestingly, IL-27 protein and transcripts of the IL-27 subunits Ebi3 and p28 were increased in C57BL/6 mice subjected to experimental airway inflammation induced by repeated intranasal treatment with the plant-derived cysteine protease papain, a prototypic allergen that was previously shown to rapidly and strongly trigger type 2 immune responses (Figures 1a and $\mathbf{b}$ ). We therefore took advantage of gene targeted mice to functionally explore the effects of IL-27 deficiency on innate lung immune cell populations in this model. As eosinophil accumulation has been demonstrated to be a characteristic feature of papain-induced airway inflammation, we first determined eosinophilia in lungs of $\mathrm{C} 57 \mathrm{BL} / 6$, Il27Ebi3 ${ }^{-1-}$ (hereinafter referred to as $\mathrm{Ebi3}^{-/-}$) and Il27p28 $28^{-1-}$ mice by flow cytometry. Interestingly, both IL-27 deficient strains after papain challenge accumulated significantly higher numbers of $\mathrm{SSC}^{\text {high }} \mathrm{SiglecF}^{+} \mathrm{CD} 11 \mathrm{~b}^{\text {high }}$ autofluorescence ${ }^{\text {low }}$ cells resembling eosinophils in their lungs compared to control mice (Figure 1c). Recently, cytokine production by ILC2 in response to epithelial-derived cytokines such as IL-33 or TSLP was shown to be vital for eosinophilia during protease allergen induced lung inflammation. $^{15,17}$ To study whether differences in the ILC2 lineage may contribute to increased eosinophilia in the absence of IL-27, we therefore next compared lung ILC2 amounts in wildtype and Il27p28- or Ebi3 deficient mice. In the steady state, lung ILC2 numbers were comparable between the three groups (Supplementary Figure 1 online). However, lung ILC2 numbers were substantially higher in both IL-27 deficient strains after challenge with papain (Figure 1d). Accordingly, IL-13 producing lung ILC2 (Figure 1e) and the concentrations of IL-5 and IL-13 in BAL (Figure 1f) are increased in the context of IL-27 deficiency compared to wild-type controls suggesting that IL-27 controls lung innate type 2 immune cell activation in vivo.

\section{IL-27 receptor signalling negatively regulates ILC2 activation in vitro and in vivo}

The IL-27 receptor complex is comprised of the more selectively expressed IL-27 R $\alpha$ chain and the ubiquitously present gp130 protein. ${ }^{27}$ Microarray or RNaseq analysis of sort purified ILC2 from spleen, ${ }^{28}$ lung $^{29}$ and gut $^{30}$ demonstrated a strong presence of IL-27 R $\alpha$ transcripts in these cells suggesting that IL-27 directly regulates ILC2 functions. To study whether IL-27 stimulation affects ILC2 directly, we therefore purified ILC2 from mice and exposed these cells in vitro to IL-27. Notably, IL-27 decreased the IL-33 + IL-7 stimulation-dependent upregulation of IL-13 and IL-5 intracellular expression (Figure 2a) and secretion (Figure 2b). Moreover, flow cytometric analysis of stimulation-dependent ILC2 proliferation by CFSE dilution assay or WST-1 proliferation assay demonstrated that IL-27 inhibited their cytokine dependent expansion (Figures 2c and d).

Having shown that IL-27 regulates ILC2 responses in vitro, we now wanted to test, whether IL-27 also regulates ILC2 functions in vivo. As we and others have shown that treatment of mice with these cytokines leads to strong proliferation and activation of ILC2 in different compartments of the body, ${ }^{14,31,32}$ we studied IL-33- and IL-25-dependent in vivo expansion of ILC2 in the absence or presence of IL-27 overexpression. To allow live imaging of ILC2 by flow cytometry or in vivo imaging, we crossed mice expressing Cre recombinase under control of the endogenous Ror $\alpha$ promoter with Rosa26-eYFP mice, in which a yellow (eYFP) fluorescent protein is expressed only after Cre-mediated excision of a loxP-flanked translational stop signal. Given that the transcription factor Ror $\alpha$ is strongly expressed in ILC2, these cells are marked by a non-reversible fluorescence signal that allows their identification and fate mapping in vitro and in vivo (Supplementary Figure 2a). To study the effects of IL-27 on the in vivo generation of ILC2, we expressed IL-33 in Rord ${ }^{C r e}$ Rosa26-eYFP reporter mice by hydrodynamic delivery of minicircle DNA as described $^{14}$ (Supplementary Figure 2b). Expectedly, IL-33 vector treatment resulted in considerably increased numbers of $\mathrm{Lin}^{-} \mathrm{eYFP}^{+} \mathrm{ICOS}^{+} \mathrm{KLRG}^{+}$ILC2 in lungs of these mice. By contrast, co-administration of IL-27 expression vector significantly decreased IL-33 dependent lung ILC2 accumulation and activation (Figure 3a). Likewise, in vivo ILC2 expansion in lungs by IL-25 vectors was strikingly inhibited in the presence of IL-27 (Supplementary Figure 2c). Similarly, IL-27 negatively regulated IL-33-and IL-25-dependent ILC2 proliferation and activation in other organs such as liver, spleen and mesenteric lymph nodes (not shown). Earlier studies have demonstrated that IL27 drives naïve Th1 differentiation and IFN- $\gamma$ production by $\mathrm{T}$ cells and NK cells. ${ }^{33,34}$ Given that IFN- $\gamma$ has lately been shown to be an inhibitor of IL-33 mediated ILC2 activation, ${ }^{35}$ we questioned whether the suppressive capacity of IL-27 on the ILC2 lineage in vivo was related to potential effects on IFN- $\gamma$. However, although IFN- $\gamma$ treatment diminished ILC2 numbers as previously demonstrated (Supplementary Figure 3a), IL-27 vector administration profoundly inhibited lung ILC2 accumulation in IFN- $\gamma$ deficient mice (Supplementary Figure 3b). Importantly, IL-10, a further cytokine implicated in the immunregulatory functions of IL-27 ${ }^{36}$ was not able to surrogate for IL-27 in a similar experimental setting (Supplementary Figure 3c) indicating that IL-27 induced IFN- $\gamma$ or IL-10 production by 


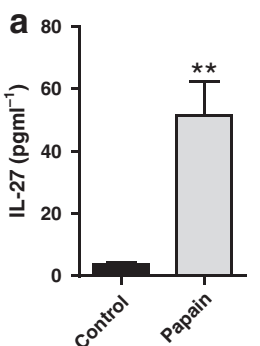

C

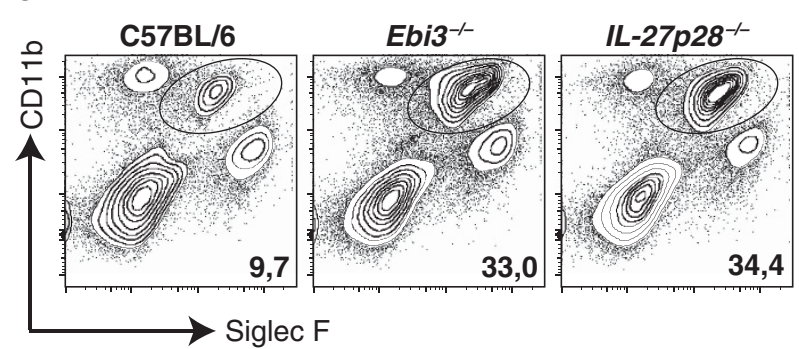

d

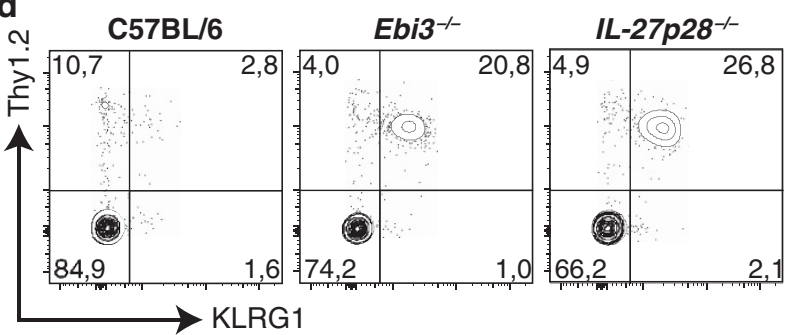

b

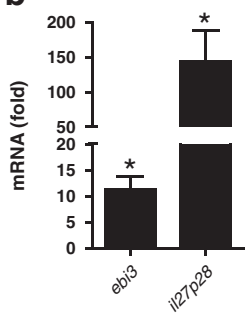

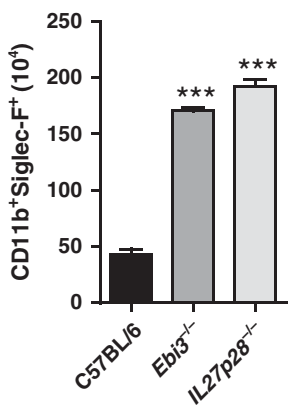
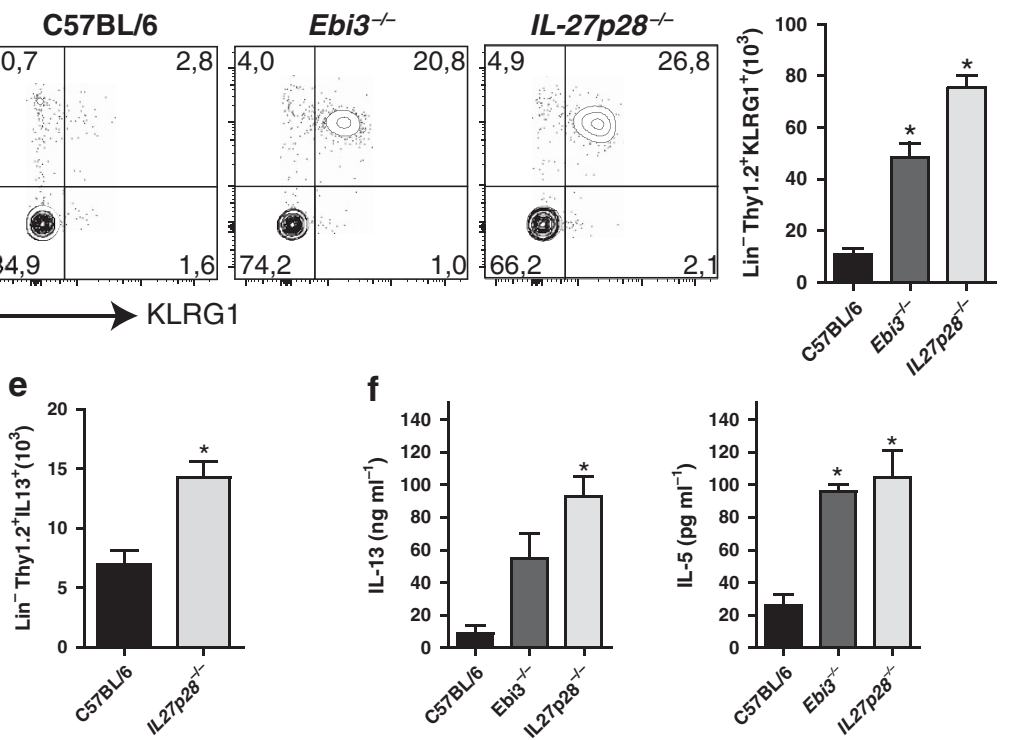

f
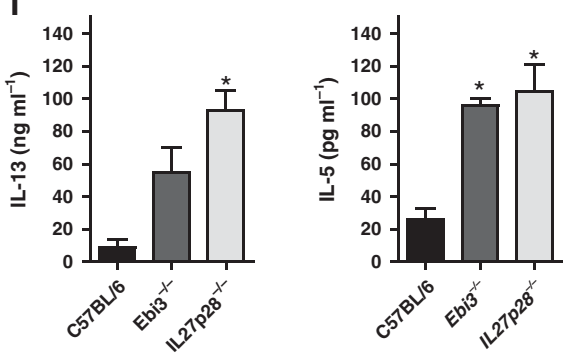

Figure 1 IL-27 deficiency is associated with increased eosinophil and ILC2 frequencies in protease allergen induced pulmonary inflammation. (a) IL-27 concentrations in control (PBS) or papain-challenged mice were determined by ELISA. (b) Total RNA was isolated from lung tissue of PBS or papaintreated mice and the expression of IL-27 subunits (Ebi3, II27p28) was analyzed by qRT-PCR relative to hprt. (c, d) Flow cytometric analysis of eosinophil $\left(\mathrm{SSC}^{\text {high }}\right.$ autofluorescence ${ }^{\text {lo }}$ SiglecF ${ }^{+} \mathrm{CD}_{11 \mathrm{~b}^{\text {high }}}$ ) and ILC2 (Lin ${ }^{-T h y} 1.2^{+} \mathrm{KLRG}^{+}$) frequencies in lungs of papain-challenged wildtype, Ebi3 ${ }^{-1-}$ and $1127 \mathrm{p} 28^{-1-}$ mice. Graphs on the right depict the quantification of absolute ILC2 numbers per organ. (e) Intracellular IL-13 expression was quantified by flow cytometry after $3 \mathrm{~h}$ stimulation with PMA and lonomycin. (f) IL-5 and IL-13 concentrations in BAL of papain-treated mice were determined by ELISA. Data represent 3 independent experiments with 4-5 mice per group. Error bars indicate s.e.m.

T cells or other immune cells does not significantly contribute to the blunted ILC2 response in the presence of IL-27. In line with this, we also observed negative regulatory effects of IL-27 on ILC2 in lymphopenic Rag1 ${ }^{-1-}$ mice (not shown).

To further determine whether intrinsic IL-27 signalling directly affects ILC2 functions, we next adoptively transferred lethally irradiated congenic C57BL/6 mice with mixed bone marrow from wildtype $(\mathrm{CD} 45.1)$ and $I L-27 \mathrm{Ra}^{-1-}$ (WSX-1 ${ }^{-1-}$, CD45.2) mice in a 1:1 ratio and studied in this setting the effects of IL-27 on IL-33 dependent ILC2 expansion in vivo. Analysis of the respective chimeras 8 weeks after reconstitution revealed that numbers of both wildtype and WSX-1 $1^{-1-}$ ILC2 were comparable in lungs and other organs 5 days after IL-33 challenge. By contrast, IL-27 treatment led only to a reduction of CD45.1 ${ }^{+}$wildtype ILC2s resulting in a selective overrepresentation of $\mathrm{WSX}-1^{+} \mathrm{CD} 45.2^{+}$cells in the total pool of ILC2 in lungs (Figure $3 \mathbf{b}$ ) and livers (Figure 3c). These data indicate that ILC2 intrinsic IL-27 signalling negatively regulates ILC2 responses in vivo. To evaluate whether IL-27 restrains ILC2 expansion during experimental lung inflammation, we next overexpressed 
a
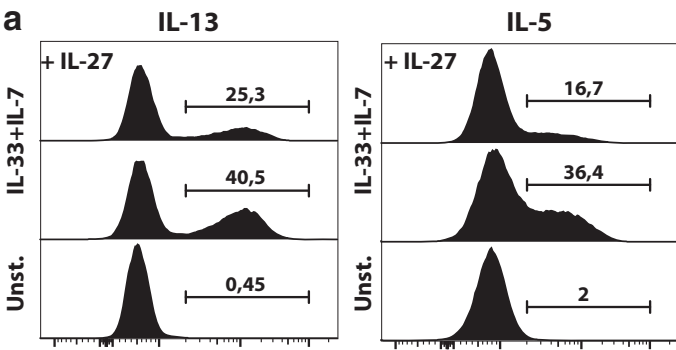

C

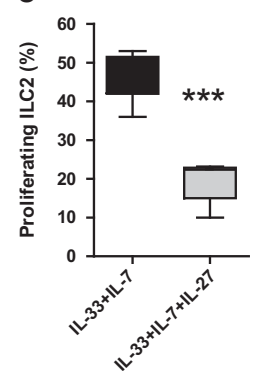

b

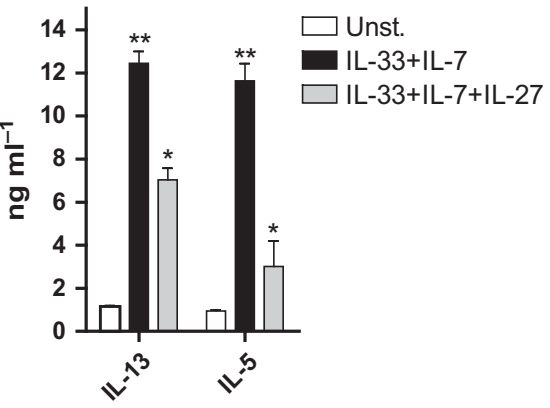

Figure 2 IL-27 regulates ILC2 activation in vitro. In vitro cultured purified ILC2 were left unstimulated (unst.) or cultured in the presence of IL-33 and IL-7 or IL-33, IL-7 and IL-27. (a) Production of IL-13 and IL-5 was determined by intracellular flow cytometry after $48 \mathrm{~h}$ of stimulation (b) IL-13 and IL-5 concentrations were determined by ELISA in supernatants obtained $24 \mathrm{~h}$ after stimulation. (c) CFSE was added to the cultures at day 0 and percentages of cells showing CFSE dilution was analyzed $72 \mathrm{~h}$ later by flow cytometry. (d) Cell proliferation was assessed with WST-1 assay at day 4 after stimulation. Data are representative of 3 different experiments $(\mathbf{a}, \mathbf{b})$ with 4 mice each or pooled $(\mathbf{c}, \mathbf{d})$ from 5 mice per group with similar results. Error bars indicate s.e.m.

IL-27 in mice subjected to papain treatment. In this setting, IL-27 profoundly reduced lung ILC2 numbers and production of IL-5 and IL-13 cytokines (Figure 3d). Moreover, IL-27 treatment diminished allergen-dependent pulmonary tissue remodeling and goblet-cell functions (Figure 3e) further indicating that IL-27 is a negative regulator of ILC2-mediated innate type 2 responses in this model.

\section{Activation of the transcription factor Stat3 blocks ILC2 responses}

IL-27 signaling to target cells is mediated by activation of the Jak-Stat pathway and selective activation of particular Stat transcription factors in responsive cells drives the cell type specific transcriptional program induced by IL-27 in these cells. Among Stats, predominantly Stat 1 and Stat 3 were shown to be selectively phosphorylated by IL-27. ${ }^{37}$ To determine which Stat proteins are activated in response to IL-27 binding to its receptor, sorted ILC2 were treated in vitro with rIL-27 and subsequently phosphorylation of Stat proteins was determined by Western blot analysis. In line with a previous report, we observed Stat1 tyrosine phosphorylation (Tyr701) in this setting (not shown). ${ }^{26}$ However, IL-27 stimulation also resulted in rapid and strong tyrosine phosphorylation of the transcription factor Stat3 (Tyr705) in ILC2 (Figure 4a). Accordingly, we next evaluated, whether forced activation of Stat3 in ILC2 affected their cytokine-mediated expansion in vivo. To this end, we overexpressed IL-33 in mice expressing a constitutively active version of Stat $3\left(\operatorname{Stat} 3 c,{ }^{38}\right)$ in ILC2 using Ror $\alpha$ Cre-mediated recombination. At day 5 after initiation of IL-33 expression, the amount of ILC2 in the lungs (Figure 4b) and livers (not shown) in $\operatorname{Ror}^{\mathrm{Cre}}{ }^{\mathrm{Stat}} 3 \mathrm{c}^{\text {flox }}$ mice was considerably lower compared to littermate controls indicating that Stat3 activation negatively regulates IL-33 dependent ILC2 accumulation. Conversely, lung ILC2 numbers in Ror $\alpha^{\text {Cre }}$ Stat $3 c^{\text {flox }}$ mice in the steady state were normal (not shown). This suggests that Stat 3 activity rather affects ILC2 activation than their developmental program. Given that IL-27 deficiency resulted in increased lung ILC2 and eosinophil numbers in papain-dependent lung inflammation, we sensitized mice reconstituted with bone marrow of Rord ${ }^{\text {Cre }}$ Stat $3 c^{\text {flox }}$ mice or littermate controls with papain and compared the accumulation of ILC2 in these mice. Ror $\alpha^{\text {Cre }}$ Stat $3 c^{\text {flox }}$ chimeric mice had strikingly fewer ILC2 and eosinophils in the lungs than controls (Figures 4c and $\mathbf{d}$ ). The inability of these mice to mount strong ILC2 responses after papain challenge was further supported by a significantly reduced presence of IL-13 producing lung ILC2 (Figure 4e). Furthermore, histological comparison of tissue sections of these groups of mice by H\&E and PAS staining showed substantially less allergen-dependent inflammatory changes and mucus production in lungs of Ror $\alpha^{\text {Cre }}$ Stat $3 c^{\text {flox }}$ mice (Figure 4f) than in the controls. In summary, these results clearly demonstrate that Stat3 activation in ILC2 protects mice from excessive type 2 mediated inflammatory lung responses. 
a

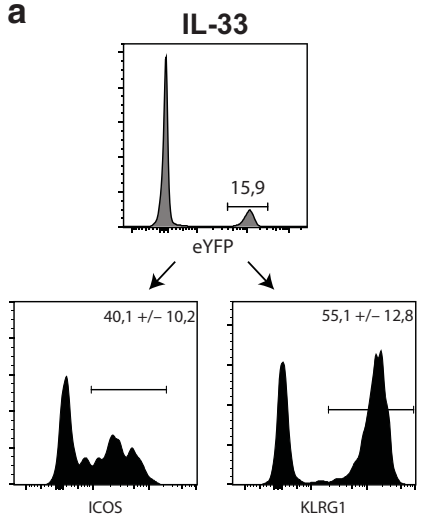

b
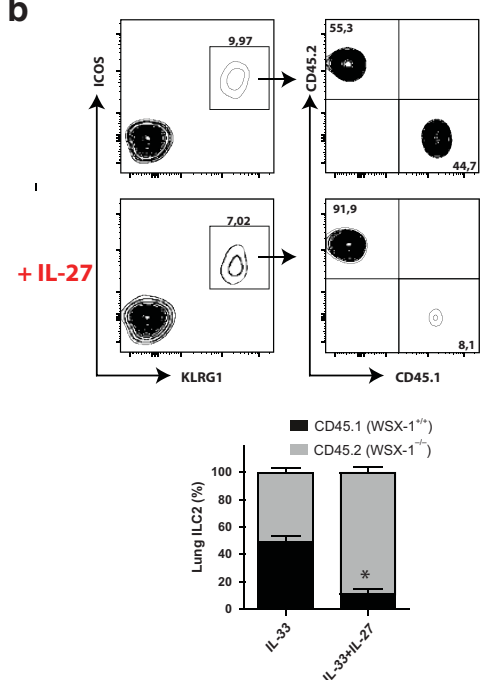

d

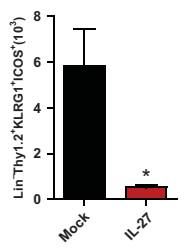

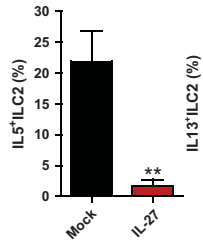

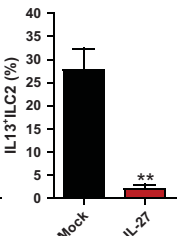

IL-33+IL-27

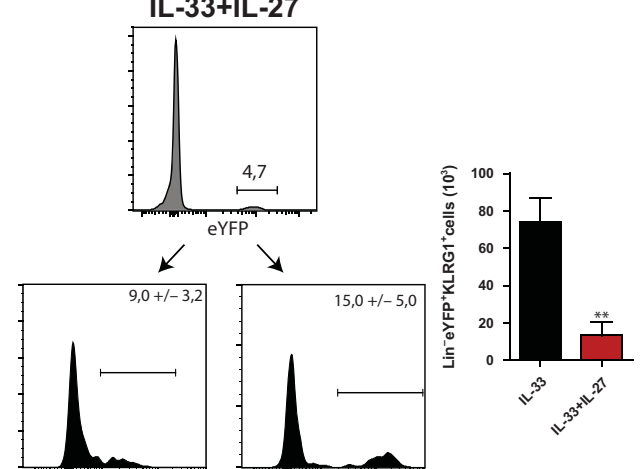

C
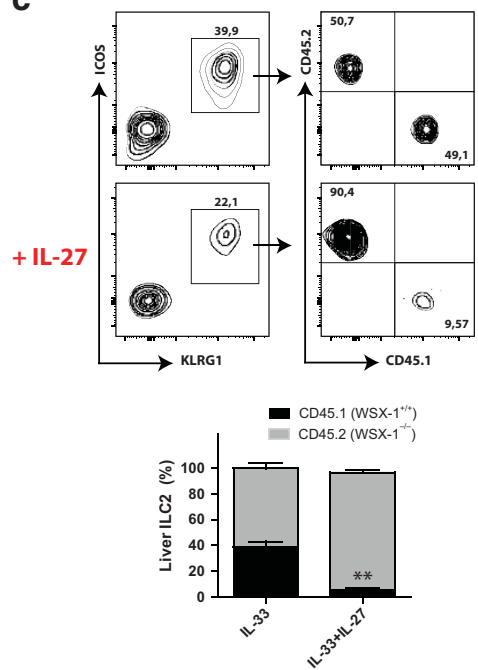

e

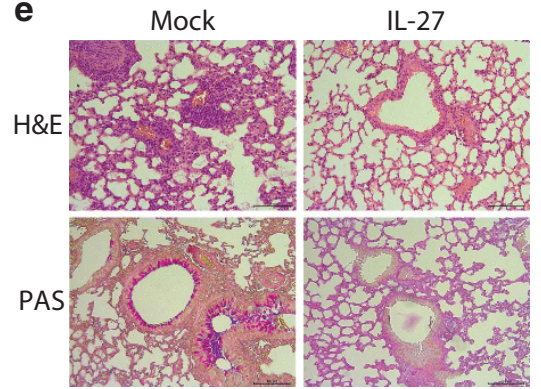

Figure 3 IL-27 suppresses ILC2 expansion in vivo. (a) Rord ${ }^{C r e}$ Rosa26-eYFP mice were injected with IL-33 vector in combination with an empty control or IL-27 vector. 5 days later LineYFP ${ }^{+}$cells in lungs were analyzed for KLRG1 and ICOS expression by flow cytometry. The bar graph depicts the quantification of absolute ILC2 numbers per organ. (b, c) Lethally irradiated wildtype mice were reconstituted with bone marrow cells isolated from C57BL/ 6 (CD45.1) or WSX-1 ${ }^{-1-}$ (CD45.2) mice in a 1:1 ratio. 8 weeks later mice were injected with IL-33 vector in combination with empty control or IL-27 vector. 5 days later immune cells in lungs (b) and livers (c) were analyzed by flow cytometry. Plots were pregated on Lin ${ }^{-}$cells. (d, e) Mock or IL-27 vector treated C57BL/6 mice were subjected to repeated intranasal papain challenges as described in the methods section. (d) Lung cells were isolated and ILC2 numbers and IL-13 and IL-5 production in gated ILC2 after restimulation with PMA/lonomycin were determined by flow cytometry. (e) Paraffin-embedded lung tissue sections were analyzed by Periodic acid-Schiff-alcian blue (PAS) staining and Hematoxylin \& Eosin (H\&E) staining. Error bars indicate s.e.m.

\section{Negative regulation of ILC2 by IL-27 directly impacts anti-parasitic immune responses in vivo}

Several previous studies in the $N$. brasiliensis model clearly indicated that ILC2 rapidly expand during helminth infections and moreover, ILC2-derived cytokines, particularly IL-13, finally drive worm clearance in the course of a primary infection. ${ }^{28,39-41}$ In order to evaluate the role of IL-27 on ILC2 functions in this model, we experimentally infected $E b i 3^{-1-}$ mice and control mice with 500 L3 stage $N$. brasiliensis larvae and enumerated ILC2 in lungs of these mice by flow cytometry. As a result, IL-27 deficieny was associated with significantly increased numbers of ILC2 in lungs (Figure 5a) and tracheal lymph nodes (tLN, Figure 5b) at day 7 post infection. Consistently, increased production of IL-5 and IL-13 was 

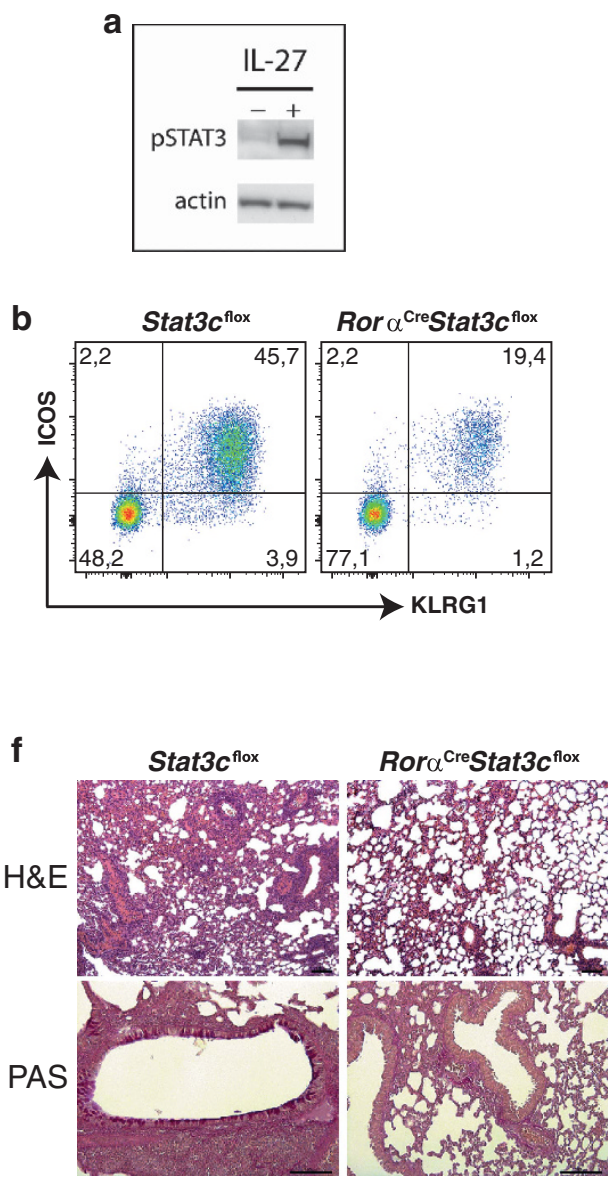

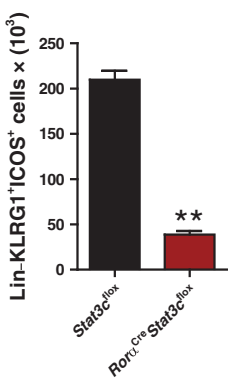

C

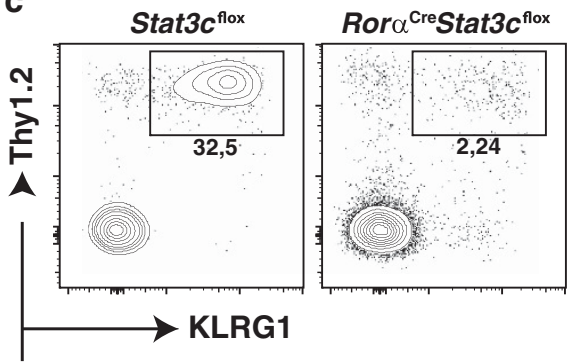

d Stat3c $c^{\text {fox }}$
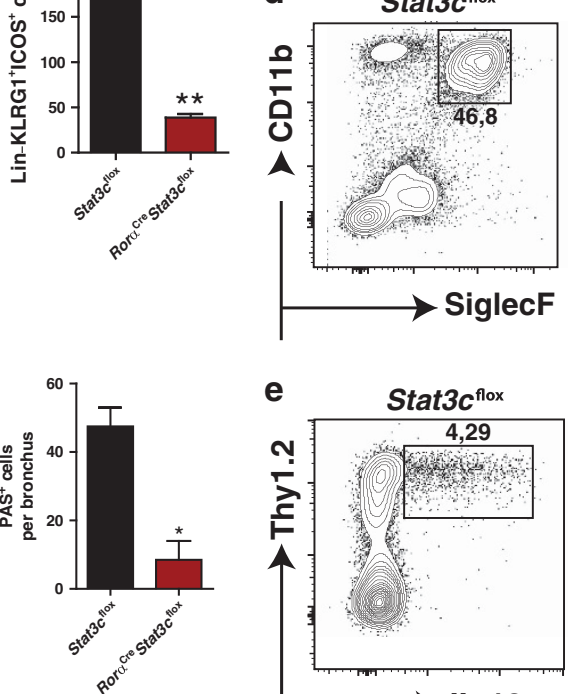

e

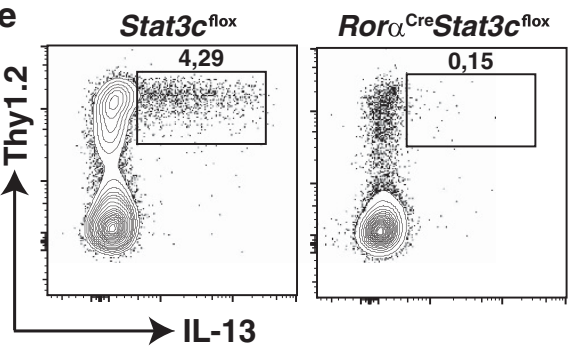

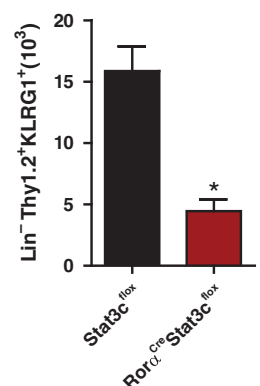

Rord $^{\text {CreStat3 }}$ c flox $^{2}$
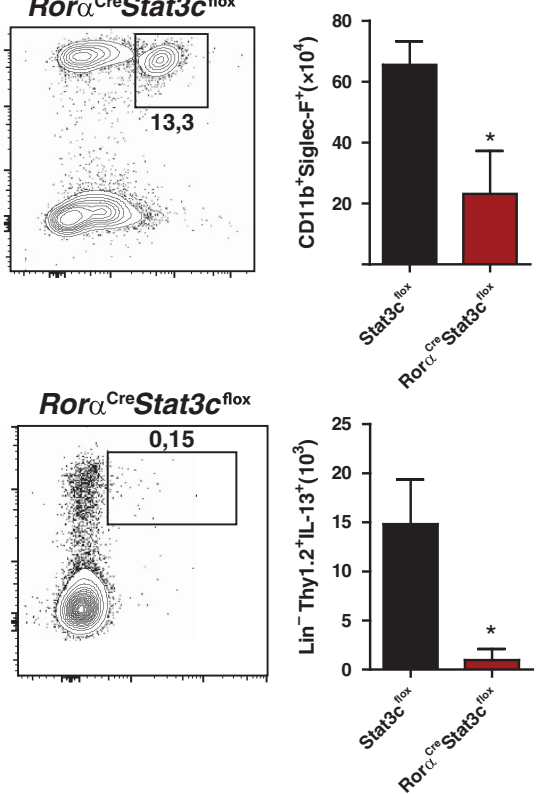

Figure 4 Conditional activation of the transcription factor Stat3 represses ILC2 activation and pulmonary inflammation. (a) Purified ILC2 were left unstimulated or were stimulated for $30^{\prime}$ with $20 \mathrm{ng} / \mathrm{ml} \mathrm{rmIL-27.} \mathrm{Whole} \mathrm{cell} \mathrm{extracts} \mathrm{were} \mathrm{prepared,} \mathrm{and} \mathrm{phospho-Stat}{ }^{\text {Y705 }}$ levels were detected by Western blot analysis. The same blot was stripped and reprobed with anti $\beta$-Actin antibodies to verify equal protein loading. (b) ILC2 were quantified by flow cytometric analysis in IL-33 vector treated control or Ror $\alpha^{\text {Cre }}$ Stat $3 \mathrm{c}^{\text {flox }}$ mice. Plots were pregated on Lin ${ }^{-T h y} 1.2^{+}$cells isolated from lungs at day 5 after IL-33 treatment. (c-f) mice of the indicated genotypes were subjected to repeated papain treatment as described in the methods section and ILC2 (c), eosinophils (d) and IL-13+ ILC2 (e) were analyzed in the lungs 1day after the last papain challenge. Bar graphs depict the quantification of absolute cell numbers per organ. (f) paraffin-embedded lung sections of papain-treated mice were analyzed by PAS staining and H\&E staining. Data represent 2 independent experiments with 5 mice per group. Error bars indicate s.e.m.

observed in sera (Figure 5c) and stimulated lung cells (Figure 5d) of infected $\mathrm{Ebi3}^{-1-}$ mice. Moreover, the increased total abundance and activation of ILC2 in these mice was associated with augmented ability to expel worms from their small intestines (Figure 5e). Notably, increased worm expulsion in $\mathrm{Ebi3}^{-l-}$ mice was accompanied by increased hyperplasia and activation of small intestinal goblet cells as indicated by enhanced PAS-staining in tissue sections (Figure 5f) and an increased transcript abundance of the goblet cell markers Retnlb and Muc5ac (Figure 5g). Moreover, we observed significantly elevated IL-13 gene expression in guts of infected $\mathrm{Ebi3}^{-1-}$ mice compared to wildtype controls suggesting that in these mice augmented ILC2 functions support antiparasitic immunity via IL-13 dependent goblet cell activation (Figure 5h). Conversely, IL-27 vector treatment of $N$. brasiliensis infected C57BL/ 6 mice led to diminished ILC2 (Figures 6a and $\mathbf{b}$ ) and eosinophil
(Figure 6c) accumulation as well as decreased IL-13 production (Figure 6d). Notably, decreased goblet cell hyperplasia in the IL-27 vector treated group (Figure 6e) was accompanied by delayed parasite clearance in these mice (Figure 6f) further supporting the concept that that IL-27-dependent negative regulation of type 2 responses impacts anti-helminthic immunity.

The presence of the transcription factor Ror $\alpha$ is required for ILC2 development and mice lacking a functional Ror $\alpha$ protein fail to expel $N$. brasiliensis worms from their guts efficiently., ${ }^{3,4}$ In order to further ascertain the functional role for IL-27 R signaling in ILC2 during helminth infections, we expressed IL-27 in lethally irradiated mice that received bone marrow from Ror $\alpha$-gene targeted mice and infected them with $N$. brasiliensis larvae. Subsequently, these mice received adoptive transfer of purified ILC2 isolated from wildtype mice or IL-27 signalling deficient WSX-1 $(I L-27 R \alpha)^{-/-}$mice 
a
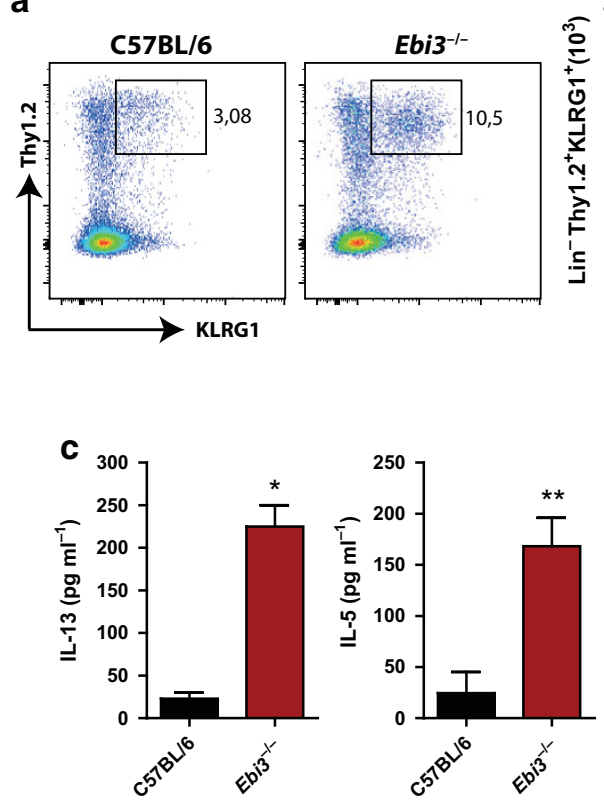

$\mathbf{f}$
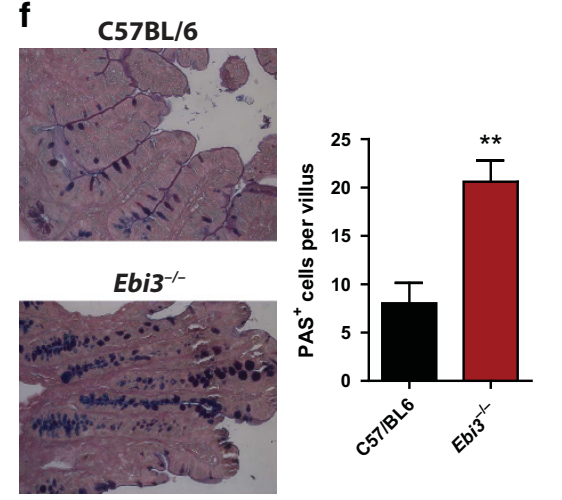

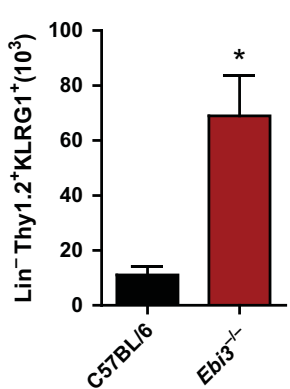

b

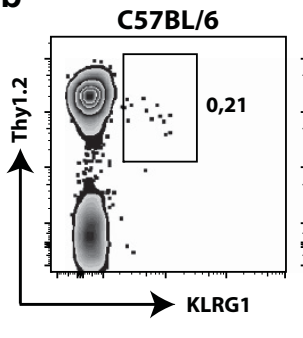

d
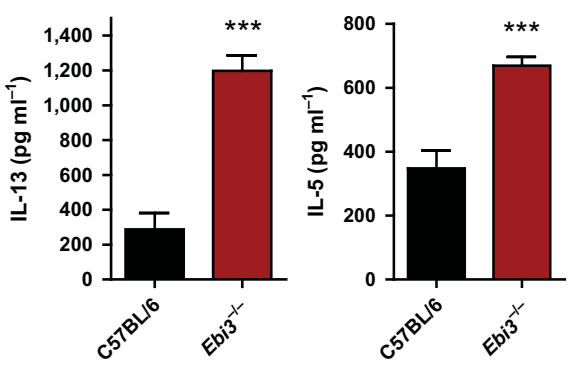

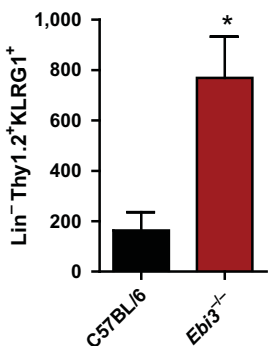

e

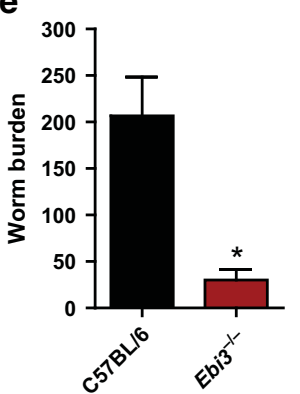

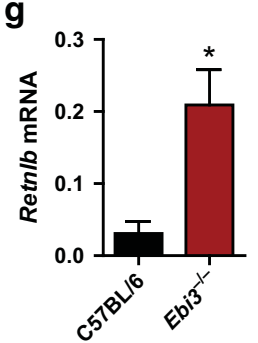
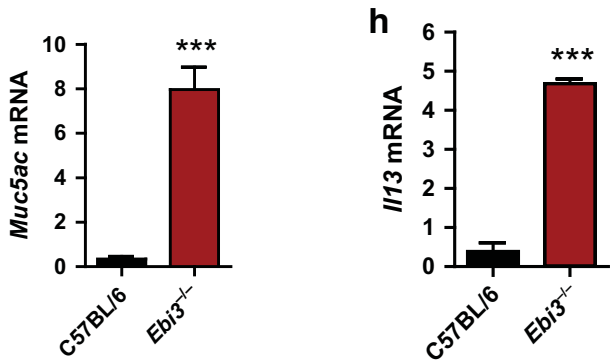

Figure 5 IL-27 as a negative regulator of ILC2-mediated anti-helminthic immunity. C57BL/6 and Ebi3 ${ }^{-1-}$ mice were infected with 500 L3 N. brasiliensis larvae and analyzed 7 d.p.i. ILC2 frequencies in lungs (a) and tLNs (b) were analyzed by flow cytometry. Plots were pregated on lin cells. Graphs on the right depict the quantification of absolute ILC2 numbers per organ. IL-5 and IL-13 concentrations in sera (c) or supernatants of PMA/lonomycin stimulated lung leukocytes (d) were determined by specific ELISAs. (e) Small intestinal worm burdens were enumerated. (f) Small intestinal tissue sections were stained with PAS and goblet cells per villus were quantified. $(\mathbf{g}, \mathbf{h})$ mRNAs of indicated genes were quantified in total RNA of small intestinal tissue lysates by qRT-PCR. Data represent 3 independent experiments with 4-5 mice per group. Error bars indicate s.e.m.

respectively. Strikingly, at day 8 post infection small intestinal worm burdens in mice that received BM of $W S X-1^{-/-}$mice were significantly lower than in control mice adoptively transferred with wildtype BM. This was accompanied by augmented hyperplasia of intestinal goblet cells (Figure $\mathbf{6 g}$ ). Collectively, these data demonstrate that regulation of ILC2 by IL-27 directly impacts anti-parasitic immune responses in vivo.

\section{DISCUSSION}

Type 2 cytokine responses have crucial host-protective functions during parasitic infections and tissue repair. Though, dysregulated type 2 immunity has been related to the pathogenesis of many allergic and fibrotic disorders. ${ }^{1}$ The type 2 inflammatory response is characterized by complex interactions of several innate cellular players including eosinophils, basophils, mast cells and alternatively activated macrophages. ${ }^{42}$ Recently, ILC2 have been demonstrated to be important early innate mediators of prototypic type 2 responses. ${ }^{11}$ They have been shown to secrete tissue repair factors like amphiregulin and regulate via constitutive and inducible production of IL-5, IL-9 and IL-13 steady state and inducible numbers of eosinophils and alternatively activated macrophages. In contrast, dysregulated ILC2 activation has been strongly implicated in chronic inflammatory immune responses that contribute to organ destruction and tissue remodeling. ${ }^{1}$ Overall, published data clearly suggest that a detailed understanding of pathways that regulate ILC2 functions may be fundamental for a broad understanding of type 2-mediated inflammatory processes at mucosal surfaces and other parts of the body. However, how factors of the immune system control ILC2 and their effector functions and maintenance is not well understood. In the present study we 

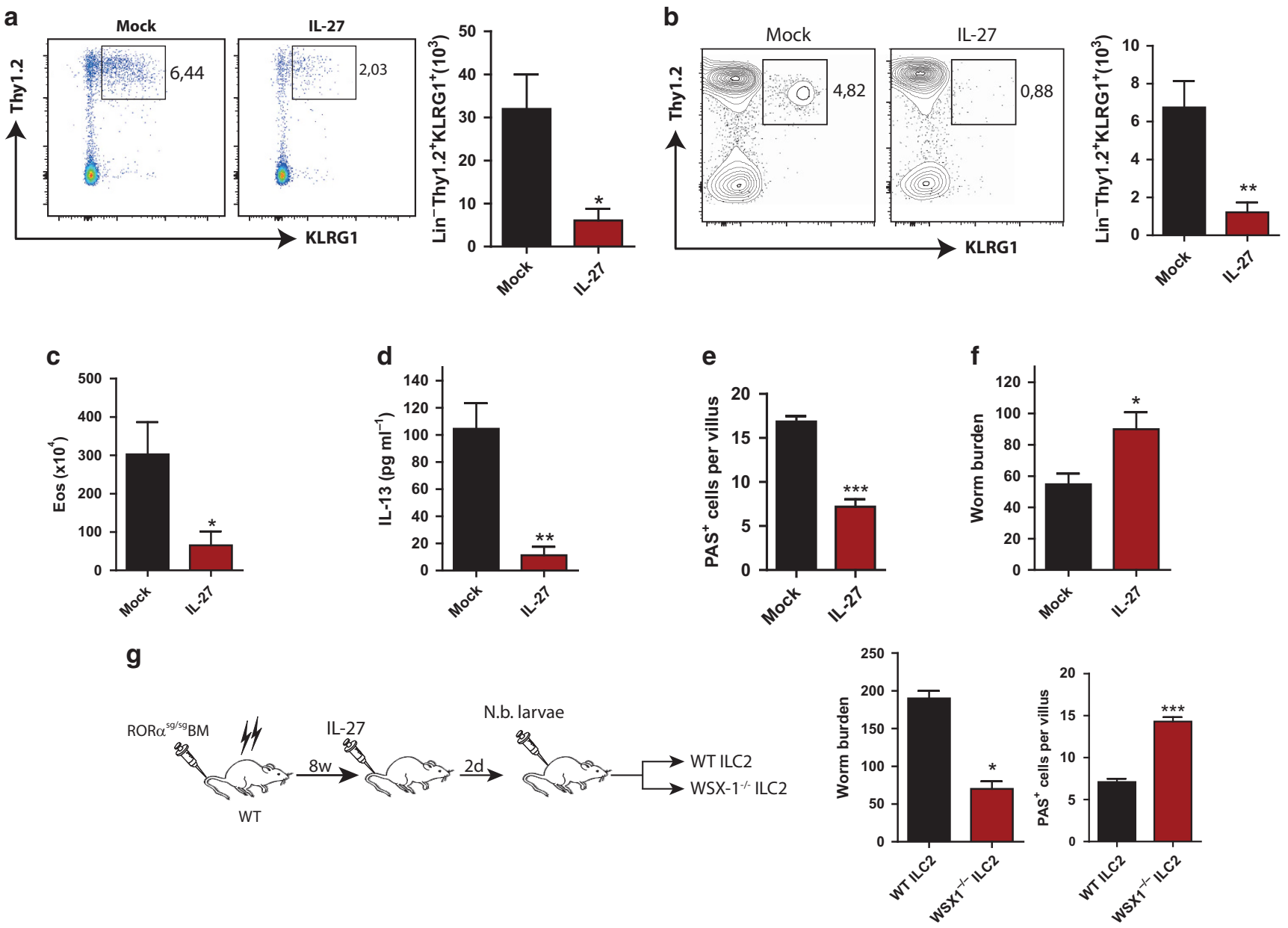

Figure 6 IL-27 effects on ILC2 impair anti-helminthic immune responses. (a-f) Mock or IL-27 vector treated C57BL/6 mice were infected with N. brasiliensis as described in the methods section and analyzed at day 9 post infection. ILC2 in lungs (a) and tLN (b) and eosinophils (c) in lungs were enumerated by flow cytometry. (d) IL-13 concentrations in sera were determined by ELISA. (e) Numbers of PAS + goblets cells were quantified in small intestinal tissue sections. (f) Small intestinal worm burdens were quantified in dissected tissues. (g) BM cells (1x $10^{7}$ ) obtained of Ror ${ }^{\text {sg/sg }}$ mice were i.v. injected into lethally irradiated C57BL/6 recipients. After $8 \mathrm{w}$, the animals were treated with IL-27 vector and infected with $N$. brasiliensis after 2 days followed by adoptive transfer of $2 \times 10^{6}$ purified WT or WSX-1 ${ }^{-1-}$ ILC2. Intestinal worm burdens in infected chimeric mice 8 d.p.i. were determined and $\mathrm{PAS}^{+}$goblet cells were quantified in small intestinal tissue sections. Data represent two different experiments with 4-5 mice per group. Error bars indicate s.e.m.

provide evidence that IL-27 receptor signalling directly regulates ILC2 responses in vivo and reveal a novel mechanism for the negative regulation of the innate arm of type 2 immune responses. We and others have previously shown that IL-27 strongly promotes Th1-mediated immunity, whereas functions of Th17 cells, induced regulatory $\mathrm{T}$ cells and some cell types of myeloid origin were diminished by this cytokine. ${ }^{19-21,43,44}$ In addition, Artis et al. established a role of IL-27 signaling in mast cells and Th2 cells that affected effective anti-helminth immunity. ${ }^{22}$ In line with this, the induction of IL-27 has been shown to limit the exacerbated Th2 responses in experimental asthma. ${ }^{24,45,46}$ Given that activated ILC2 not only trigger the early inflammatory response, but also contribute to Th2 cell differentiation via IL-13 production $^{47}$ and MHCII/TCR interaction, ${ }^{48}$ those findings may be at least partially related to IL-27 inhibitory effects on ILC2 that we describe here. The molecular mechanism of the suppressive effect of IL-27 on
ILC2 remains to be fully determined. Recent reports indicated that IFN- $\gamma$, type I IFNs and IL-27 suppress ILC2 via Stat1 activation. ${ }^{25,26}$ We observed in addition to Stat 1 activation also rapid Stat 3 phosphorylation in ILC2 in vitro in response to IL-27 and Stat3 overactivation in ILC2 in transgenic mice prevented their efficient cytokine-dependent accumulation in vivo. IL-27 was previously shown to antagonize Th2 cells by regulation of the major transcriptional regulator Gata3. ${ }^{49}$ Moreover, Stat3 has been implicated in IL-27 dependent Gata3 suppression in mucosal FoxP3 ${ }^{+}$Treg $^{50}$ and Stat3 activation reduced Gata3 expression in T cells. ${ }^{51}$ ILC2 development and functions critically depend on Gata3, suggesting that a similar mechanism may also account for the negative regulation of ILC2 by IL-27-dependent Stat1 or Stat3 activation. Interestingly, forced activation of Stat 3 in ILC2 by expressing a constitutively active form under regulation of the Rora gene promoter profoundly diminished protease induced allergic 
inflammation further indicating that suppression of ILC2 by manipulation of common signalling pathways is sufficient to prevent disease development. By contrast, activation of Stat 5 by factors such as IL-2, IL-7 and TSLP has been connected to enhanced Gata3 activity and type 2 cytokine production in ILC2. ${ }^{18,52}$ Further studies including mice with conditional inactivation of particular Stat factors in ILC2 are required to fully understand the molecular signalling events in ILC2 that are activated by IL-27 receptor signalling.

Previous work demonstrated that the Th1 signature cytokine IFN- $\gamma$ crossregulates Th2 responses and IL-27 induction of IFN- $\gamma$ expression in T cells and natural killer cells (NK) cells has been implicated in its suppressive capacity. ${ }^{37}$ While we provide multiple lines of evidence for a functional ILC2 intrinsic IL-27 receptor signalling, Molofsky et al. found in a recent study that IFN $-\gamma$ profoundly inhibited ILC2 cytokine production and cellular proliferation. ${ }^{35} \mathrm{We}$ demonstrate here that IL-27 efficiently suppressed ILC2 accumulation in IFN- $\gamma^{-1-}$ mice indicating that IL-27 may counterregulate ILC2 by multiple mechanisms. Similarly to IL-27, IFN- $\gamma$ administration diminished eosinophilia and delayed expulsion of adult worms in the N. brasiliensis model. ${ }^{53}$ However, we did not measure increased IFN- $\gamma$ levels after IL-27 injection in both $N$. brasiliensis infected and papain-treated mice. Moreover, in the context of IL-27 deficiency, IFN- $\gamma$ concentrations were not reduced in both models (not shown) indicating that in these models, IL-27 induced IFN- $\gamma$ does not largely contribute to the regulatory effects of IL-27 on ILC. However, the IL-27/IFN- $\gamma /$ ILC axis may be more relevant in settings, where IL-27 dependent IFN- $\gamma$ induction is critical for disease control or manifestation. Although gene expression data indicate that ILC2 express the IL-10 receptor chains ${ }^{30}$ and IL-10 primarily mediates its suppressive functions via Stat $3,{ }^{54}$ our data indicated that IL-27 induction of IL-10 e.g. by Tr1 cells is also not substantially relevant in these settings.

Collectively, our in vitro and in vivo findings provide clear evidence that IL-27 receptor signalling directly regulates ILC2 responses and reveal a novel mechanism for the negative regulation of the innate arm of type 2 immune responses. Given that ILC2 are increasingly recognized as innate cell population that drives central features of type 2 responses in vivo, our data here extend the knowledge about their regulation in vivo and about the pleiotropic immunoregulatory functions of IL-27. Thus, therapeutic modulation of the IL-27/IL-27 R $\alpha$ axis may be relevant in a number of human inflammatory conditions associated with dysregulated type 2 responses.

\section{MATERIAL AND METHODS}

Animals. C57BL/6, IFN- $\gamma^{-1-}$, Rosa26Stop-eYFP mice, Rag1 ${ }^{-/-}$and $W S X-1^{-1-}$ mice were originally obtained from Jackson Laboratories. Ror $^{\text {sg/sg }}{ }^{\text {Rora }}{ }^{\text {tmla(EUCOMM)Wtsi }}$ ) were from EUCOMM. Ror ${ }^{\text {Cre }}{ }^{\text {mice }}$ were kindly provided by Dennis O'Leary. ${ }^{55}$ Il27Ebi3 $^{-1-}$ and Il27p28 $8^{-1-}$ were on C57BL/6 background. Rosa26Stat3 $c^{\text {stopfl/fl }}$ mice were obtained from S. Koralov. ${ }^{38}$ Bone marrow of Rora $^{\mathrm{Cre}} \mathrm{R}$ osa26Stat $3 c^{\text {stopfl/fl }}$ mice or littermate controls was adoptively transferred to lethally irradiated ( $10 \mathrm{~Gy}) \mathrm{C} 57 \mathrm{BL} / 6$ recipient mice. In all experimental procedures, 8- to 12-week-old background, age- and sex-matched mice were used. Ror $\alpha^{\mathrm{sg} / \mathrm{sg}}$ mice were used shortly after weaning to generate BM chimeras. Animal experiments were approved by the governments of Rheinland-Pfalz and Mittelfranken.

In vivo expression of cytokines. The generation of IL-25 and IL-33 minicircle-based vectors was described previously by us. ${ }^{14}$ Similarly, an expression cassette encoding for a bioactive single-chain EBI3:p28 heterodimer was cloned in to the SpeI and SalI sites of pMC-Hep. DNAs were isolated with Qiagen Plasmid-Maxikits including endotoxin removal. Subsequently, the DNA was treated with Miraclean endotoxin Removal Kits (MirusBio Madison). 2,5-10 $\mu$ g of DNA were administrated in Krebs-Ringer solution to mice via hydrodynamic tail vein injection. In all treated mice, serum concentrations of the relevant cytokines were deterimend by ELISA to monitor successful administration.

Antibodies and flow cytometry. Tissue cell suspensions from different mouse organs were incubated with anti-Fc receptor blocking antibody (anti-CD16/CD32, eBioscience) prior to staining with specific antibodies. The cells were stained with combination of the FITC-, PE-, APC-, PerCP-Cy5.5- fluorochrome-tagged antibodies and biotin-conjugated monoclonal antibodies (all from eBioscience unless specified otherwise). Streptavidin conjugated to Brilliant Violet 421, $\mathrm{PE}$, FITC were purchased from BioLegend. The following antibodies were used for flow cytometry: anti-Thy1.2 (53-2.1), anti-KLRG1 (2F1), anti-ICOS (7E.17G9), anti-IL-13 (eBio13A), anti-IL-5 (TRFK5), antiGata3 (TWAJ), anti-Gr-1 (RB6-8C5), anti-Siglec-F (ES22-10D8, Miltenyi Biotec), anti-CD11b (M1/70), anti-CD3e (145-2C11), anti-CD45R/B220 (RA3-6B2), anti-Ter-119 (Ter119). A premixed lineage cocktail containing biotinylated anti-CD3, anti-CD5, anti-CD11b, anti-B220, anti-NK1.1, anti-Ter-119, anti-Gr1, and anti-Siglec-F mAbs was obtained from Miltenyi Biotec. Samples were measured on an LSRFortessa cell analyzer (BD Bioscience) and were analyzed by using Flowjo (Treestar).

ILC2 isolation and culture. Immune cells were isolated from lung, liver, lymph nodes or spleen with the gentleMacs device according to manufacturer's instructions (Miltenyi Biotec).

For generation of large ILC2 numbers for in vitro studies, ILC2 were expanded in vivo by treatment of mice with IL-33 as described previously. ${ }^{14} 5$ days later ILC2 were purified from spleens and MLN by magnetic separation using a combination of biotinylated antibodies including CD3e, CD5, CD11b, CD11c, CD45R, Ly-6B.2, Ter119, Siglec-F, NK1.1 and Gr-1 followed by incubation with anti-biotin microbeads. Further purification was achieved by FACS using a FACSAria II device in the Core Unit Cell Sorting Erlangen.

Assessment of cell proliferation. Purified ILC2 were stimulated with $10 \mathrm{ng} / \mathrm{ml}$ rIL-33 (ImmunoTools) and rIL-7 (Miltenyi Biotec) in combination with $20 \mathrm{ng} / \mathrm{ml}$ rIL27 (BioLegend) for $72 \mathrm{~h}$, WST-1 proliferation reagent (Roche) was added (1:10 dilution) and incubated at $37^{\circ} \mathrm{C}$. The absorbance was measured at $450 \mathrm{~nm}$. Alternatively, ILC2 were labeled with $5 \mu \mathrm{M}$ CFSE (Invitrogen), stimulated with the aforementioned recombinant proteins for $72 \mathrm{~h}$ and CFSE dilution was measured by flow cytometry.

Helminth infection protocol. Mice strains were subcutaneously inoculated with 500 viable L3 larvae of $N$. brasiliensis. Mice were maintained on water containing $1 \mathrm{mg} / \mathrm{mL}$ of sulfadoxin and $0.2 \mathrm{mg} / \mathrm{mL}$ of trimethoprim for $5 \mathrm{~d}$ and sacrificed and euthanized at day 7-9. Tissue was collected for further analysis (histology, qRT-PCR, flow cytometry) and worm burdens in small intestines were enumerated in dissected tissue under a dissecting microscope (Olympus SZX7). In some experiments, wildtype mice were treated with IL-27 vector injection five days prior to infection.

Papain induced airway-inflammation. Mice were anesthetized with isoflurane and exposed intranasally to $50 \mu \mathrm{g}$ papain (Merck) in $20 \mu \mathrm{l}$ 
PBS on days $0,1,2$ and analyzed on day 3 . Alternatively, $25 \mu \mathrm{g}$ papain was inhaled on day 0,3 and 6; 7 d later mice were re-challenged and the experiment was analyzed at day 17 . Serum was collected and stored at $-20{ }^{\circ} \mathrm{C}$ for further analysis. In some experiments, wildtype mice were treated with IL-27 vector five days prior to papain challenge.

Bone marrow chimeras. For generation of bone marrow chimeras, bone marrow cells $\left(1 \times 10^{7}\right)$ obtained from femurs and tibias of donor wildtype, WSX-1 ${ }^{-1-}$ or Ror $\alpha^{s g / s g}$ mice were i.v. injected into lethally irradiated (10 Gy) C57BL/ 6 recipient mice. After 8 weeks, the animals were injected with IL-27 vector after two days and infected with $N$. brasiliensis followed by adoptive transfer of $2 \times 10^{6}$ purified wildtype or WSX-1 $1^{-1-}$ ILC2. The experiment was analyzed 8 d.p.i.

ELISA. For determination of mouse IFN- $\gamma$, IL-5, IL-10, IL-13, IL-25, IL-27 and IL-33 concentrations in sera and cell culture supernatants specific ELISA Kits from BioLegend and eBioscience were used according to manufacturer's instructions.

Gene expression analysis. Total RNA from snap-frozen tissues was isolated with RNeasy mini kits according to the manufacturer's instructions (Qiagen). cDNA was generated using the Script RT-PCR kit from Jenabioscience (Jena, Germany). qRT-PCR analysis was performed using QuantiTect Primer assays from Qiagen in a CFX96 system (Bio-Rad). Relative differences between samples were calculated using hprt as reference gene.

Statistical analysis. Student's $t$-test or Mann-Whitney U tests were performed with Graphpad 6 software (Prism). $P$-values $<0.05$ with a $95 \%$ confidence interval were considered significant.

SUPPLEMENTARY MATERIAL is linked to the online version of the paper at http://www.nature.com/mi

\section{ACKNOWLEDGMENTS}

This research has received funding from DFG projects within SFB796 (B7), SPP1656, KFO257 and SFB1181 (to S.W and M.F.N). S.W. and T.M. were supported by the Interdisciplinary Center for Clinical Research (IZKF) of the University Erlangen-Nuremberg. The authors thank D. Beß, C. Lindner, A. Taut, M. Wagner and C. Weiss for excellent technical assistance.

\section{AUTHOR CONTRIBUTIONS}

T.M. conducted the majority of the experiments and analysed data; M.K. did experiments; A.T and D.V. supported the $N$. brasiliensis infection experiments. M.F.N provided ideas and contributed to the writing of the manuscript; S.W. designed and coordinated the study and wrote the paper.

\section{DISCLOSURE}

The authors declared no conflict of interest.

c) 2016 Society for Mucosal Immunology

\section{REFERENCES}

1. McKenzie, A.N., Spits, H. \& Eberl, G. Innate lymphoid cells in inflammation and immunity. Immunity 41, 366-374 (2014).

2. Diefenbach, A., Colonna, M. \& Koyasu, S. Development, differentiation, and diversity of innate lymphoid cells. Immunity 41, 354-365 (2014).

3. Halim, T.Y. et al. Retinoic-acid-receptor-related orphan nuclear receptor alpha is required for natural helper cell development and allergic inflammation. Immunity 37, 463-474 (2012).

4. Wong, S.H. et al. Transcription factor RORalpha is critical for nuocyte development. Nat Immunol 13, 229-236 (2012).

5. Hoyler, T. et al. The transcription factor GATA-3 controls cell fate and maintenance of type 2 innate lymphoid cells. Immunity 37, 634-648 (2012).

6. Klein Wolterink, R.G. et al. Essential, dose-dependent role for the transcription factor Gata3 in the development of IL-5 + and IL-13+ type 2 innate lymphoid cells. Proc Natl Acad Sci U S A 110, 10240-10245 (2013).
7. Spooner, C.J. et al. Specification of type 2 innate lymphocytes by the transcriptional determinant Gfi1. Nat Immunol 14, 1229-1236 (2013).

8. Yu, Y. et al. The transcription factor Bcl11b is specifically expressed in group 2 innate lymphoid cells and is essential for their development. J Exp Med 212, 865-874 (2015).

9. Walker, J.A. et al. Bcl11b is essential for group 2 innate lymphoid cell development. J Exp Med 212, 875-882 (2015).

10. Califano, D. et al. Transcription Factor Bcl11b Controls Identity and Function of Mature Type 2 Innate Lymphoid Cells. Immunity 43, 354-368 (2015).

11. von Moltke, J. \& Locksley, R.M. I-L-C-2 it: type 2 immunity and group 2 innate lymphoid cells in homeostasis. Curr Opin Immuno/ 31, 58-65 (2014).

12. Koyasu, S. \& Moro, K. Th2-type innate immune responses mediated by natural helper cells. Ann N Y Acad Sci 1283, 43-49 (2013).

13. Licona-Limon, P. et al. Th9 Cells Drive Host Immunity against Gastrointestinal Worm Infection. Immunity 39, 744-757 (2013).

14. McHedlidze, T. et al. Interleukin-33-dependent innate lymphoid cells mediate hepatic fibrosis. Immunity 39, 357-371 (2013).

15. Halim, T.Y., Krauss, R.H., Sun, A.C. \& Takei, F. Lung natural helper cells are a critical source of Th2 cell-type cytokines in protease allergen-induced airway inflammation. Immunity 36, 451-463 (2012).

16. Christianson, C.A. et al. Persistence of asthma requires multiple feedback circuits involving type 2 innate lymphoid cells and IL-33. J Allergy Clin Immunol 136, 59-68.e14 (2015).

17. Turner, J.E. et al. IL-9-mediated survival of type 2 innate lymphoid cells promotes damage control in helminth-induced lung inflammation. $J$ Exp Med 210, 2951-2965 (2013).

18. Roediger, B. et al. IL-2 is a critical regulator of group 2 innate lymphoid cell function during pulmonary inflammation. J Allergy Clin Immunol 136, 1653-1663.e7 (2015).

19. Neufert, C. et al. IL-27 controls the development of inducible regulatory T cells and Th17 cells via differential effects on STAT1. Eur J Immunol 37, 1809-1816 (2007).

20. Wirtz, S. et al. Protection from lethal septic peritonitis by neutralizing the biological function of interleukin 27. J Exp Med 203, 1875-1881 (2006).

21. Stumhofer, J.S. et al. Interleukin 27 negatively regulates the development of interleukin 17-producing T helper cells during chronic inflammation of the central nervous system. Nat Immunol 7, 937-945 (2006).

22. Artis, D. et al. The IL-27 receptor (WSX-1) is an inhibitor of innate and adaptive elements of type 2 immunity. J Immunol 173, 5626-5634 (2004).

23. Miyazaki, Y. et al. Exacerbation of experimental allergic asthma by augmented Th2 responses in WSX-1-deficient mice. J Immunol 175, 2401-2407 (2005).

24. Yoshimoto, T., Yoshimoto, T., Yasuda, K., Mizuguchi, J. \& Nakanishi, K. IL-27 suppresses Th2 cell development and Th2 cytokines production from polarized Th2 cells: a novel therapeutic way for Th2-mediated allergic inflammation. J Immunol 179, 4415-4423 (2007).

25. Moro, K. et al. Interferon and IL-27 antagonize the function of group 2 innate lymphoid cells and type 2 innate immune responses. Nat Immunol 17, 76-86 (2016).

26. Duerr, C.U. etal. Type I interferon restricts type 2 immunopathology through the regulation of group 2 innate lymphoid cells. Nat Immunol 17, 65-75 (2016).

27. Pflanz, S. et al. WSX-1 and glycoprotein 130 constitute a signaltransducing receptor for IL-27. J Immunol 172, 2225-2231 (2004).

28. Neill, D.R. et al. Nuocytes represent a new innate effector leukocyte that mediates type-2 immunity. Nature 464, 1367-1370 (2010).

29. Motomura, Y. et al. Basophil-derived interleukin-4 controls the function of natural helper cells, a member of ILC2s, in lung inflammation. Immunity 40 , 758-771 (2014).

30. Robinette, M.L. et al. Transcriptional programs define molecular characteristics of innate lymphoid cell classes and subsets. Nat Immunol 16, 306-317 (2015).

31. Huang, Y. et al. IL-25-responsive, lineage-negative KLRG1(hi) cells are multipotential 'inflammatory' type 2 innate lymphoid cells. Nat Immuno/ 16, 161-169 (2015).

32. Saenz, S.A. et al. IL-25 simultaneously elicits distinct populations of innate lymphoid cells and multipotent progenitor type 2 (MPPtype2) cells. J Exp Med 210, 1823-1837 (2013). 
33. Villarino, A. et al. The IL-27R (WSX-1) is required to suppress T cell hyperactivity during infection. Immunity 19, 645-655 (2003).

34. Owaki, T. et al. A role for IL-27 in early regulation of Th1 differentiation. $J$ Immunol 175, 2191-2200 (2005).

35. Molofsky, A.B. et al. Interleukin-33 and Interferon-gamma CounterRegulate Group 2 Innate Lymphoid Cell Activation during Immune Perturbation. Immunity 43, 161-174 (2015).

36. Yoshida, H. \& Hunter, C.A. The immunobiology of interleukin-27. Annu Rev Immunol 33, 417-443 (2015).

37. Hunter, C.A. \& Kastelein, R. Interleukin-27: balancing protective and pathological immunity. Immunity 37, 960-969 (2012).

38. Mesaros, A. et al. Activation of Stat3 signaling in AgRP neurons promotes locomotor activity. Cell Metab 7, 236-248 (2008).

39. Price, A.E. et al. Systemically dispersed innate IL-13-expressing cells in type 2 immunity. Proc Natl Acad Sci U S A 107, 11489-11494 (2010).

40. Hung, L.Y. et al. IL-33 drives biphasic IL-13 production for noncanonical Type 2 immunity against hookworms. Proc Natl Acad Sci U S A 110, 282 287 (2013).

41. Oeser, K., Schwartz, C. \& Voehringer, D. Conditional IL-4/LL-13-deficient mice reveal a critical role of innate immune cells for protective immunity against gastrointestinal helminths. Mucosal immunology 8, 672-682 (2015).

42. Paul, W.E. \& Zhu, J. How are $T(H) 2$-type immune responses initiated and amplified?. Nat Rev Immunol 10, 225-235 (2010).

43. Pflanz, S. et al. IL-27, a heterodimeric cytokine composed of EBI3 and p28 protein, induces proliferation of naive CD4 + Tcells. Immunity 16, 779-790 (2002).

44. Mascanfroni, I.D. et al. IL-27 acts on DCs to suppress the T cell response and autoimmunity by inducing expression of the immunoregulatory molecule CD39. Nat Immunol 14, 1054-1063 (2013).
45. Dokmeci, E. et al. EBI3 deficiency leads to diminished Thelper type 1 and increased Thelper type 2 mediated airway inflammation. Immunology 132 , 559-566 (2011).

46. Fujita, H. et al. Production of both IL-27 and IFN-gamma after the treatment with a ligand for invariant NK T cells is responsible for the suppression of Th2 response and allergic inflammation in a mouse experimental asthma model. J Immunol 183, 254-260 (2009).

47. Halim, T.Y. et al. Group 2 innate lymphoid cells are critical for the initiation of adaptive T helper 2 cell-mediated allergic lung inflammation. Immunity 40 , 425-435 (2014).

48. Oliphant, C.J. et al. MHCll-mediated dialog between group 2 innate lymphoid cells and CD4(+) T cells potentiates type 2 immunity and promotes parasitic helminth expulsion. Immunity 41, 283-295 (2014).

49. Lucas, S., Ghilardi, N., Li, J. \& de Sauvage, F.J. IL-27 regulates IL-12 responsiveness of naive CD4 + T cells through Stat1-dependent and independent mechanisms. Proc Natl Acad Sci U S A 100, 15047-15052 (2003).

50. Wohlfert, E.A. et al. GATA3 controls Foxp3(+) regulatory T cell fate during inflammation in mice. J Clin Invest 121, 4503-4515 (2011).

51. Yang, X.O. et al. STAT3 regulates cytokine-mediated generation of inflammatory helper T cells. J Biol Chem 282, 9358-9363 (2007).

52. Mjosberg, J. et al. The transcription factor GATA3 is essential for the function of human type 2 innate lymphoid cells. Immunity 37, 649-659 (2012).

53. Urban, J.F. Jr. et al. IFN inhibits inflammatory responses and protective immunity in mice infected with the nematode parasite, Nippostrongylus brasiliensis. J Immunol 151, 7086-7094 (1993).

54. Murray, P.J. Understanding and exploiting the endogenous interleukin-10/ STAT3-mediated anti-inflammatory response. Curr Opin Pharmacol 6, 379-386 (2006).

55. Chou, S.J. et al. Geniculocortical input drives genetic distinctions between primary and higher-order visual areas. Science 340, 1239-1242 (2013). 